\title{
Astrocyte-Derived Thrombospondin-2 Is Critical for the Repair of the Blood-Brain Barrier
}

\author{
Weiming Tian, Andrew Sawyer, \\ Feriha B. Kocaoglu, and Themis R. Kyriakides \\ From the Vascular Biology and Therapeutics Program, the \\ Departments of Patbology and Biomedical Engineering, Yale \\ University, New Haven, Connecticut
}

Thrombospondin (TSP)-2-null mice have an altered brain foreign body response (FBR) characterized by increases in inflammation, extracellular matrix deposition, and leakage of the blood-brain barrier (BBB). In the present study, we investigated the role of TSP-2 in BBB repair during the brain FBR to mixed cellulose ester filters implanted in the cortex of wild-type (WT) and TSP-2-null mice for 2 days to 8 weeks. Histological and immunohistochemical analysis revealed enhanced and prolonged neuroinflammation in TSP-2-null mice up to 8 weeks after implantation. In addition, recovery of the BBB was compromised and was associated with increased gelatinolytic activity and low levels of collagen type IV in the basement membranes of TSP-2-null blood vessels. An analysis of protein extracts from implantation sites revealed elevated levels of matrix metalloproteinase (MMP)-2 and MMP-9 in TSP-2-null brains. TSP-2-null astrocytes secreted higher levels of both MMPs in vitro compared with their WT counterparts. Furthermore, TSP-2-null astrocytes were deficient in supporting the recovery of barrier function in WT endothelial cells. Finally, Western blot analysis of astrocytes and brain endothelial cells revealed TSP-2 expression only in the former. Taken together, our observations suggest that astrocyte-derived TSP-2 is critical for the maintenance of physiological MMP-2 and MMP-9 levels during the FBR and contributes to the repair of the BBB. (Am J Pathol 2011, 179:860-868; DOI: 10.1016/j.ajpath.2011.05.002)

The long-term biocompatibility of implantable devices is critical for their continued function in the brain. The trauma from device insertion, glial scar formation, and long-term inflammation combine to erode implantable electrode responses. ${ }^{1,2}$ We recently described the brain foreign body response (FBR) in mice deficient in matrix metalloproteinase (MMP)-9 and showed prolonged leakage of the blood-brain barrier (BBB). ${ }^{3}$ Previously, we also showed that MMP-2 and MMP-9 levels and extracellular matrix (ECM) remodeling were altered in thrombospondin (TSP)-2-null mice during various injury models, including wound healing, ischemia, and the FBR. ${ }^{4}$ TSP-2 is a matricellular protein with antiangiogenic activity that influences ECM assembly via modulation of MMP levels. ${ }^{5}$ Moreover, TSP-2-null mice display increased levels of soluble vascular endothelial growth factor in wounds, which is consistent with enhanced neovascularization. ${ }^{6}$ In addition, the inhibition of TSP-2 expression during wound healing by localized antisense gene delivery resulted in altered ECM remodeling, increased angiogenesis, and accelerated healing. ${ }^{7}$ Taken together, these observations suggest that TSP-2 deposition in damaged tissues influences critical processes during repair. Furthermore, studies ${ }^{8}$ have indicated an association between TSP-2 and vascular integrity. For example, prolonged and enhanced vascular leakage was observed during skin inflammation in TSP-2-null mice. Moreover, leaky vessels found in Akt1-null animals because of TSP-1 and TSP-2 deficiencies were recovered by reexpression of TSP-2. ${ }^{9}$ However, little is known about the role of TSP-2 in the function of the BBB or the effects of foreign body implants on the BBB.

In a previous study, ${ }^{10}$ we characterized the fibrovascular invasion of polyvinyl alcohol sponges implanted in the brain cortex of TSP-2-null and wild-type (WT) mice. Although these implants are substantially larger than traditional microelectrodes, they provide a clear brain-biomaterial interface for histological examination. Histological and immunohistochemical (IHC) analyses of the implanted sponges revealed minimal invasion at 4 weeks and robust invasion at 8 weeks after implantation. Sponges implanted in TSP-2-null mice displayed significantly higher levels of invasion at 8 weeks compared with those implanted in WT mice. Moreover, TSP-2-null

\footnotetext{
Supported by NIH grant GM-072194.

Accepted for publication May 2, 2011

Address reprint requests to Themis R. Kyriakides, Ph.D., Yale University School of Medicine, Amistad Research Bldg, 10 Amistad St, Room 301C, PO Box 208089, New Haven, CT 06520. E-mail: themis. kyriakides@yale.edu.
} 
mice displayed enhanced levels of tissue-associated serum albumin, an indicator of BBB leakage.

To further investigate the role of TSP-2 in the repair of the BBB and the brain FBR in general, we implanted mixed cellulose ester filters in the cortex of WT and TSP2-null mice and analyzed the ensuing response from 2 days up to 8 weeks. The choice of material, cellulose ester, was made so that the implant could be in place during tissue processing and sectioning. This preserved the interface between the implant and brain tissue and, thus, allowed for the retention of surface-adjacent cells. We believe this was important because we previously demonstrated the presence of multiple cell layers on retrieved brain implants. ${ }^{11}$ In the present study, we show that TSP-2 is critical for the repair of the BBB during the FBR. Furthermore, in vitro studies show that astrocytes are a major source of TSP-2. This is in agreement with previous studies ${ }^{12,13}$ that have shown that TSP-2 is expressed by immature astrocytes in the central nervous system and promotes the formation of synapses. In addition, our studies show that TSP-2 is critical for the repair of damaged BBB via the modulation of MMP levels.

\section{Materials and Methods}

\section{Animal Models}

A total of 70 TSP-2-null and 70 WT mice, both in a mixed C57BL/6 $\times 129$ Svj genetic background, were used for all experiments in a procedure approved by the Institutional Animal Care and Use Committee at Yale University (New Haven, CT). Cortical implantations were performed as previously described. ${ }^{3}$ Before surgery, mice were anesthetized with a ketamine and xylazine cocktail (100 and $10 \mathrm{mg} / \mathrm{kg}$ i.p., respectively) and immobilized in a head holder (model 921-E; David Kopf Instruments, Tujunga, CA). Subsequently, a midline scalp incision exposed the skull; and a 2.0-mm burr hole was made using a variablespeed drill (Ideal Microdrill; Harvard Apparatus, Holliston, MA) at $1.0 \mathrm{~mm}$ posterior to the bregma and $1.0 \mathrm{~mm}$ to the right of the midline. A sterile piece of Millipore filter (2- $\mathrm{mm}^{2}$ mixed-cellulose ester) was implanted into the cortex. These filters were removed at different periods, ranging from 2 days to 8 weeks, as previously described. ${ }^{3}$ At 2 weeks after implantation, 16 TSP-2 knockout (KO) mice were given either a $40 \mathrm{mg} / \mathrm{kg}$ dose i.p. of the MMP inhibitor (GM6001; US Biologicals, Marblehead, MA) dissolved in dimethyl sulfoxide (at $10 \mathrm{mg} / \mathrm{mL}$ ) or dimethyl sulfoxide alone (vehicle control) every 3 days for 2 weeks. After the 2 weeks of treatment, animals were perfused and processed for histological analysis, as described later.

\section{Brain Tissue Processing}

Mice were transcardially perfused with PBS ( $30 \mathrm{~mL}, 0.1$ $\mathrm{mol} / \mathrm{L}$ ), followed by $4 \%$ paraformaldehyde ( $25 \mathrm{~mL}$ in PBS) at either 2 days or $1,2,4$, or 8 weeks after implantation. Before perfusion, mice were anesthetized with an i.p. injection of a ketamine-xylazine cocktail. The brain was removed and postfixed overnight in $4 \%$ paraformalde- hyde in PBS at $4^{\circ} \mathrm{C}$. After fixation, the tissue was allowed to equilibrate in a solution of $30 \%$ sucrose in PBS at $4^{\circ} \mathrm{C}$ for 24 to 48 hours. Tissues were embedded in paraffin, and coronal sections were generated across the implant site. Selected tissues were processed without fixation and embedded in OCT compound (optimal cutting temperature; Tissue-Tek; Sakura, Torrance, CA) to generate $10-\mu \mathrm{m}$ cryosections.

\section{Histochemistry and IHC}

Paraffin-embedded sections (5- $\mu \mathrm{m}$ thick) were deparaffinized and rehydrated before histological evaluation and IHC. H\&E staining was performed according to standard protocols. IHC detection of TSP-1, MMP-9, and F4/80 required antigen retrieval by immersion in citrate buffer $(\mathrm{pH} 6)$ for 30 minutes at $100^{\circ} \mathrm{C}$. A mouse-on-mouse staining kit (Vector Labs, Burlingame, CA) was used to detect TSP-1 (ab1823 used at a dilution of 1:25; Abcam (Cambridge, MA). To confirm the ability of this antibody to specifically detect mouse TSP-1, bone marrow sections were stained to detect megakaryocytes (data not shown). For von Willebrand factor detection, antigen retrieval was performed by treatment of sections with proteinase $\mathrm{K}$ for 10 minutes at $37^{\circ} \mathrm{C}$. TSP-2 was detected as previously described. ${ }^{10}$ Primary antibodies were diluted with $1 \%$ bovine serum albumin in PBS and blocked overnight at $4^{\circ} \mathrm{C}$. Biotinylated secondary antibodies against rabbit, goat, or rat IgGs were used at 1:200 dilutions. All immunoperoxidase reactions were performed using the avidinbiotin method, with $3^{\prime}, 3^{\prime}$-diaminobenzidine as a chromogen and a methyl green counterstain on all slides.

\section{MSA Extravasation Studies}

The extent of mouse serum albumin (MSA) extravasation into brain tissue was determined at 2 days to 8 weeks after implantation. The sections were stained with antibodies to mouse serum albumin, as previously described. ${ }^{14}$ Six high-power images (original magnification, $\times 400$ ) were obtained from each site at a distance up to $100 \mu \mathrm{m}$ from the implant, and 12 images per genotype per time point were used for quantification. Digital images were analyzed using image analysis software (Metamorph; Molecular Devices, Sunnyvale, CA).

\section{Protein Extraction and Zymography}

Fragments of lateral cortex and striatum were extracted to assess the presence of gelatinolytic activity, as described by Zhang and Gottschall. ${ }^{15}$ Briefly, $0.25 \mathrm{~g}$ of tissue was homogenized in $0.7-\mathrm{mL}$ lysis buffer supplemented with detergents [Brij-35 (Sigma Chemical, St. Louis, MO) and 1\% Triton X-100 (American Bioanalytical, Natick, MA)]. Protein content was determined by Bradford assay, and samples were analyzed using Western blot analysis, according to standard protocols. In addition, a portion of the homogenate was incubated with gelatin-Sepharose 4B (Pharmacia, Peapack, NJ) for 1 hour at $4^{\circ} \mathrm{C}$ and centrifuged for 10 minutes at $8,600 \times \mathrm{g}$. Pellets were resuspended in $150 \mu \mathrm{L}$ of elution buffer, supplemented with $10 \%$ 
dimethyl sulfoxide for 30 minutes at $4^{\circ} \mathrm{C}$. Samples were subjected to zymography in $8 \%$ polyacrylamide gels containing $1 \mathrm{mg} / \mathrm{mL}$ porcine gelatin. For detection of gelatinase activity in situ, cryosections of brain tissues were incubated with DQ gelatin (Invitrogen, Eugene, OR) and analyzed by fluorescence microscopy, as previously described. ${ }^{16} \mathrm{Im}$ ages (original magnification, $\times 400$ ) were taken from each site at a distance up to $100 \mu \mathrm{m}$ from the implant.

\section{Brain Endothelial Cell Isolation}

Mouse brain microvascular endothelial cells (mBMECs) were prepared as previously described, with minor modifications. ${ }^{17}$ Briefly, 4- to 6-week-old mice were euthanized, the brains were removed, and the cerebral cortices were excised, separated from the white matter and leptomeninges, and minced in HBSS (Invitrogen Corp, Carlsbad, CA) with the aid of a scalpel blade. Samples were then treated with $1 \%$ collagenase type I for 2 hours at $37^{\circ} \mathrm{C}$. The homogenates were suspended in $20 \%$ bovine serum albumin in Dulbecco's modified Eagle's medium solution (Invitrogen) and centrifuged at $1000 \times g$ at $4^{\circ} \mathrm{C}$. The supernatants were carefully aspirated; and the pellets were resuspended in HBSS, layered over a preformed Percoll gradient (Pharma$\mathrm{cia}$ ), and centrifuged at $1661 \times \mathrm{g}$ for 11 minutes. After centrifugation, the top layer containing microvessels was collected, washed once with HBSS, and then digested in HBSS solution containing $1 \mathrm{mg} / \mathrm{mL}$ collagenase-dispase (Roche, Indianapolis, IN), $10 \mathrm{U} / \mathrm{mL}$ DNase I (Sigma Chemical), and $1 \mathrm{mg} / \mathrm{mL}$ Na-p-tosyl-L-lysine chloromethyl ketone (Sigma Chemical) for 40 minutes at $37^{\circ} \mathrm{C}$. mBMECs were cultured in six-well plates coated with collagen type IV (BD Bioscience, San Jose, CA) in Dulbecco's modified Eagle's medium supplemented with $20 \%$ inactivated fetal calf serum, $2.5 \mathrm{mg} / \mathrm{mL}$ heparin (Sigma Chemical), $20 \mathrm{mmol} / \mathrm{L}$ HEPES, $2 \mathrm{mmol} / \mathrm{L}$ glutamine, antibiotic-antimycotic (Invitrogen Corp), and EC growth supplement (BD Bioscience). This protocol typically produced primary EC cultures that are approximately $97 \%$ to 99\% pure, as determined by immunocytochemistry with anti-von Willebrand factor antibody (BD Bioscience) (data not shown).

\section{Astrocyte Isolation and Cell Culture}

Cerebral astrocyte cultures were prepared from 1-dayold mice according to the method of Hertz et al, ${ }^{18}$ with minor modifications. After dissection, cerebral cortices were digested with $1 \mathrm{U} / \mathrm{mL}$ trypsin (Invitrogen) and 10 $\mathrm{U} / \mathrm{mL}$ DNAse I (Sigma Chemical) for 15 minutes at $37^{\circ} \mathrm{C}$ in HBSS. Cells were washed in HBSS, resuspended in complete astrocyte media (Dulbecco's modified Eagle's medium with $10 \%$ heat-inactivated fetal bovine serum, glutamine, and antibiotic-antimycotic), and seeded in a $75-\mathrm{cm}^{2}$ tissue culture flask. Two weeks after the initial plating, flasks were shaken for 18 hours at $37^{\circ} \mathrm{C}$; and the media, which contained predominantly neurons, were removed. Adherent cells were $>99 \%$ astrocytes, as judged by glial fibrillary acidic protein (GFAP) immunocytochemistry (data not shown). For all experiments, either first- or second-passage astrocytes were used.

\section{Brain Endothelial Barrier Function Measurements}

To establish co-cultures, $1 \times 10^{5}$ WT or TSP-2-null astrocytes were plated on the reverse side of a 12-mmdiameter insert transwell with a $0.4-\mathrm{mm}$ pore size (Transwell; Costar Corning Inc., Corning, NY). Cells were allowed to attach for 6 hours, and the inserts were turned over and placed in 24-well transwell plates. A total of $2.5 \times 10^{5}$ WT mBMECs were seeded on the upper (cell-free) side of the transwell, which was coated with $10 \mathrm{mg} / \mathrm{mL}$ fibronectin (BD Bioscience). Co-cultures were maintained at $37^{\circ} \mathrm{C}$ under normoxic conditions. Electrical resistance of established co-cultures was measured with an Endohom-12 electrical resistance apparatus (World Precision Instruments Inc., Sarasota, FL). The resistance of fibronectin-coated transwells was subtracted to calculate the final transepithelial electrical resistance values (expressed in $\Omega / \mathrm{cm}^{2}$ ). Selected wells were treated with $10 \mu \mathrm{mol} / \mathrm{L}$ MMP inhibitor. Three wells per time point, per group, were measured, and the experiment was repeated three times.

\section{EC Permeability Measurement}

To measure permeability changes, MBMEC growth medium was replaced at confluency with HBSS; $20 \mu \mathrm{L}$ of $0.5 \% \mathrm{w} / \mathrm{v}$ Evans blue dye was added to the upper chamber, and the cells were treated with $1 \mathrm{ng} / \mathrm{mL}$ lipopolysaccharide (LPS; Sigma Chemical). Samples were collected from the lower wells at 0, 5, 10, and 15 minutes, and the amount of Evans blue dye was estimated by spectrophotometry at $600 \mathrm{~nm}$. The media on the top and bottom of the chamber were replaced with HBSS before the addition of Evans blue dye at each point. Selected wells were treated with $10 \mu \mathrm{mol} / \mathrm{L} \mathrm{MMP}$ inhibitor. Three wells per time point, per group, were measured; and the experiment was performed twice.

\section{Results}

\section{TSP-2 Deposition during the Brain FBR}

IHC analysis of filters implanted in WT mice for 2 days to 8 weeks was performed to investigate the spatiotemporal distribution of TSP-2 in the brain FBR. In addition, WT brain sections without implants and TSP-2-null brain sections served as controls. TSP-2 was not detected in either control WT or TSP-2-null tissue. In contrast, TSP-2 deposition was increased after implantation in WT tissue and was predominantly associated with astrocytes within the brain parenchyma at 2 and 4 weeks (Figure 1). TSP-2 could not be detected at 8 weeks. Unlike TSP-2, we could not induce TSP-1 in the brain FBR at any point (representative images of 2- and 4-week tissues are shown in Figure 1). Occasionally, TSP-2 deposition was also detected in close proximity to blood vessels, but it was unclear if ECs were the source of TSP-2. To further investigate the cellular source of TSP-2, Western blot analysis of the conditioned media from isolated astrocytes and 

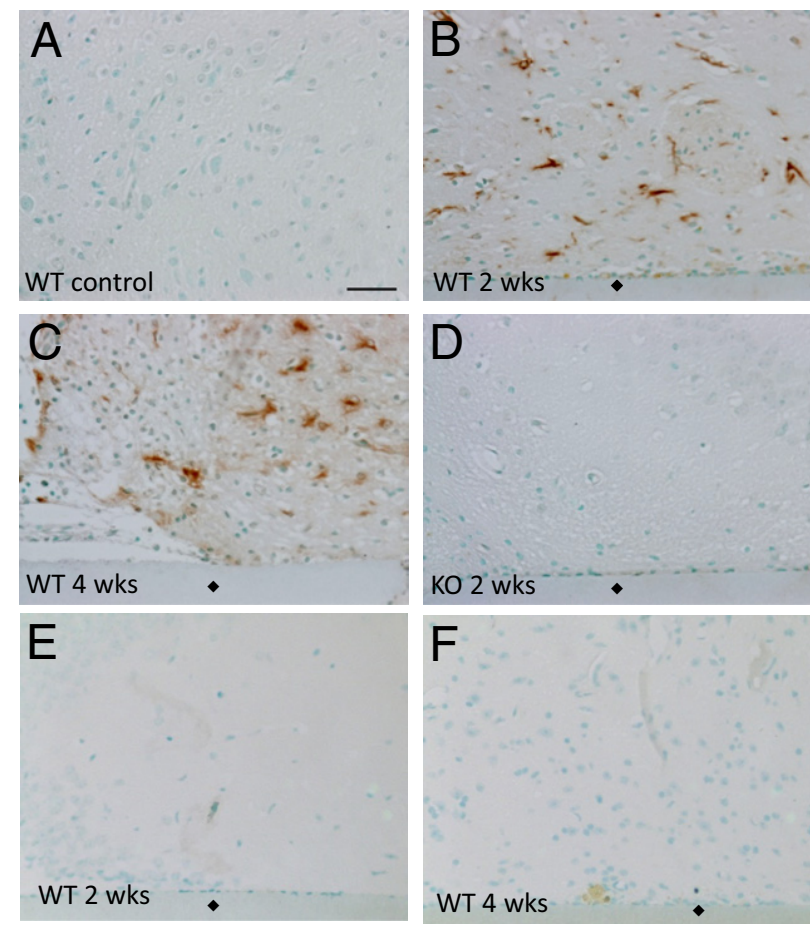

KO

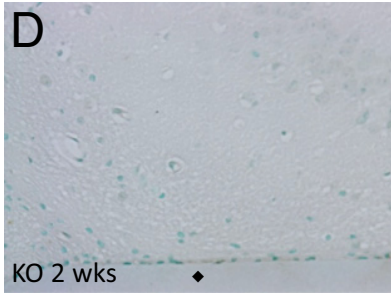

WT 4 wks

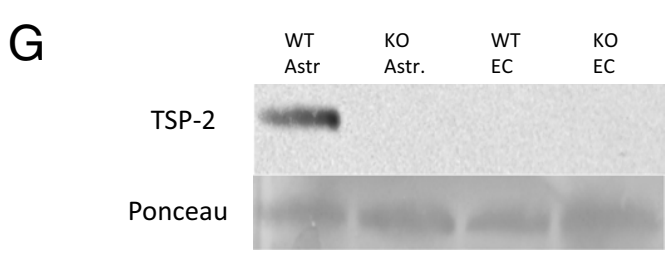

Figure 1. Increased TSP-2 deposition after material implantation. TSP-2 is detected at elevated levels in WT tissue at 2 weeks $(\mathbf{B})$ and 4 weeks (C) after implantation, when compared with WT control (A) and KO (D) mice. TSP-1 is not induced in the FBR at 2 weeks $(\mathbf{E})$ and 4 weeks $(\mathbf{F})$. G: Western blot analysis showed increased TSP-2 is associated with astrocytes (Astr). Scale bar $=100 \mu \mathrm{m}$. Diamonds indicate implant.

mBMECs was performed. Immunoreactive protein was only detected in WT astrocyte-conditioned medium, suggesting that astrocytes are the major source of TSP-2 in the brain FBR (Figure 1E).

\section{Prolonged FBR in TSP-2-Null Mice}

Previously, we showed increased neuroinflammation within the interstices of polyvinyl alcohol sponges implanted in TSP-2-null mice for 8 weeks. ${ }^{10}$ To investigate changes in the progression of neuroinflammation, the presence of macrophages/microglia was analyzed with F4/80 antibody IHC (Figure 2). Microglia/macrophage is a major glial cell type involved in the brain's inflammatory response, constituting $5 \%$ to $10 \%$ of the total number of glial cells in the brain. ${ }^{1}$ The number of positive cells per high-power field indicated the increased presence of macrophages/microglia in TSP-2-null mice as early as day 2 . The WT mice displayed a pattern of increasing macrophage/microglia accumulation from 2 to 14 days, after which the number of positive cells decreased progressively up to 8 weeks. In contrast, the presence of macrophages/microglia in TSP-2-null mice remained high throughout the duration of the experiment, suggesting persistent neuroinflammation. The increased presence of F4/80-positive cells around the implant suggests the induction of a prolonged inflammatory response.

\section{Enhanced Astrocyte Reaction in TSP-2-Null Mice}

GFAP IHC was performed to detect reactive astrocytes and to determine the extent of reactive gliosis, the term used to describe the activation, hypertrophy, and proliferation of astrocytes in response to injury. The presence of reactive astrocytes at implantation sites was evident in both WT and TSP-2-null mice. However, estimation of the GFAP-positive percentage area revealed that it was significantly higher in TSP-2-null mice at 2 days. Consistent with our findings obtained using the F4/80 antibody, the levels of GFAP immunoreactivity in WT mice peaked at 2 weeks and then decreased from 2 to 8 weeks. TSP-2-null mice had more reactive astrocytes at day 2 than WT mice, and the levels remained elevated up to 8 weeks (Figure 3). Collectively, our observations suggest that TSP-2-null mice are compromised in their ability to resolve neuroinflammation and gliosis.

\section{Blood Vessel Formation and Function in the Brain FBR}

The increased neuroinflammation in TSP-2-null mice at 4 and 8 weeks prompted us to evaluate the function of the

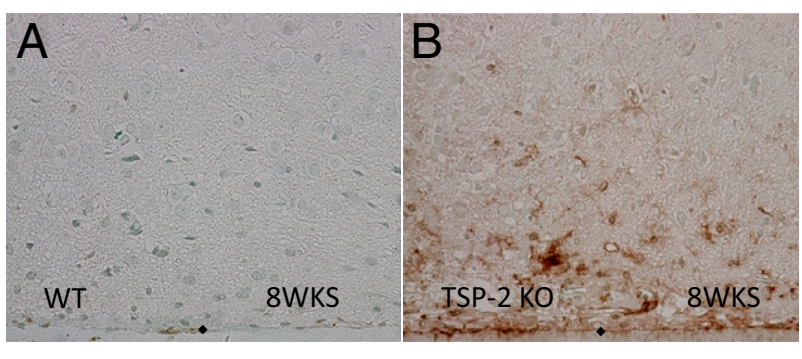

C

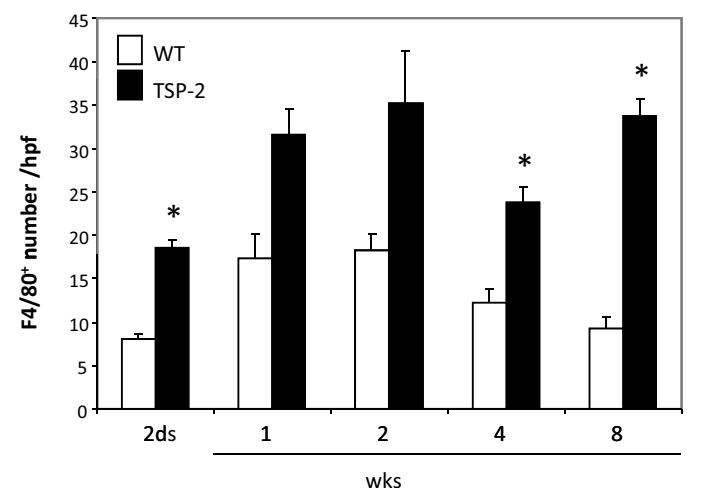

Figure 2. Increased neuroinflammation in TSP-2-null mice. IHC stain for macrophages/microglia 8 weeks after implantation. The numbers of macrophages/microglia are increased in TSP-2 KO mice (B), when compared with WT mice (A). Diamonds indicate implant. C: The number of macrophages/ microglia increased in WT mice until 2 weeks and then declined; the amount remained elevated in TSP-2 KO mice $\left({ }^{*} P<0.05\right)$. ds indicates days; hpf, high-power field 

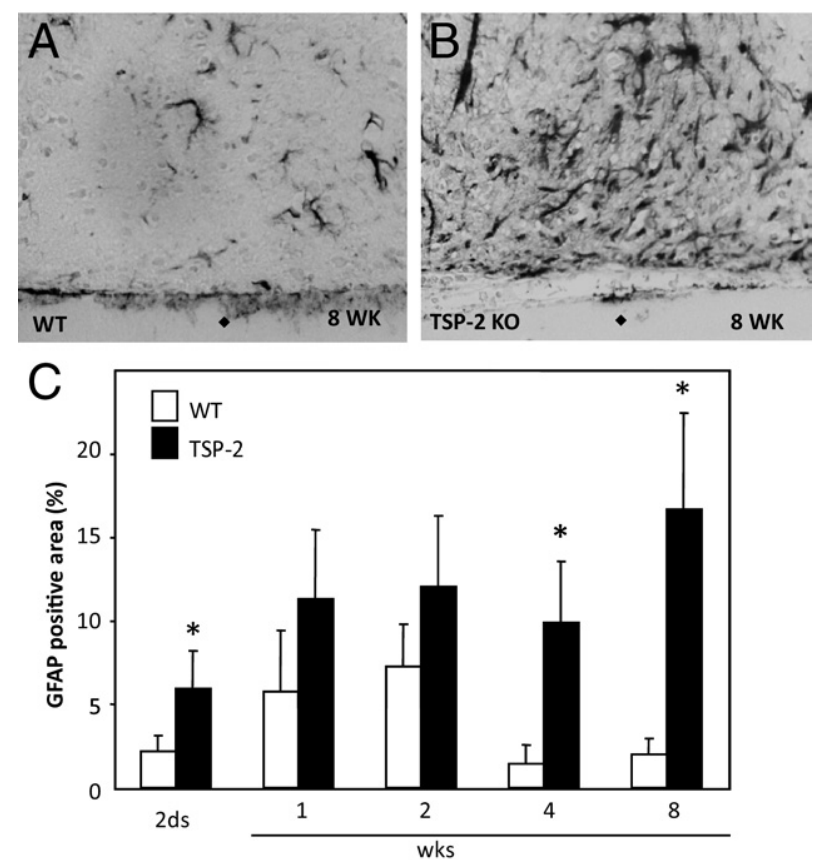

Figure 3. Increased gliosis in TSP-2-null mice. Reactive astrocytes were increased in TSP KO mice after implantation. GFAP immunoreactivity in representative tissue sections showed increased levels of reactive astrocytes in TSP KO mice (B) when compared with WT mice (A) at 8 weeks. Diamonds indicate implant (A and B). C: Reactive astrocytes peaked at 2 weeks in WT mice, whereas they remained elevated at 8 weeks in TSP-2 KO mice $\left({ }^{*} P<0.05\right)$. ds indicates days.

BBB. We postulated that the presence of a leaky BBB after filter implantation could explain the prolonged neuroinflammatory response in TSP-2-null mice. Thus, we analyzed the integrity of the BBB by detecting MSA, which should be absent from the brain parenchyma. Consistent with this requirement, we did not detect extravasation of MSA in the brains of WT and TSP-2 KO mice that did not receive implants, indicating BBB integrity (data not shown). In addition, no immunoreactivity was detected in the cerebral cortex contralateral to the implant site, indicating a structurally intact BBB at sites away from implantation (Figure 4). In contrast, high levels of MSA immunoreactivity were detected at 2 days after implantation in WT and TSP-2-null mice (Figure 4, C and D). The WT mice displayed a progressive decrease in MSA immunoreactivity from 2 days to 8 weeks. In contrast, TSP2-null mice displayed the persistent presence of MSA, suggesting continuous leakage of the BBB (Figure 4G).

$I \mathrm{HC}$ analysis of endothelial tight junction and basement membrane proteins was pursued to investigate the nature of the BBB disruption. Consistent with the antiangiogenic activity of TSP-2, von Willebrand factor $\mathrm{IHC}$, followed by enumeration of blood vessels, revealed that their number near implants was increased in TSP-2-null mice at 2 weeks (Figure 5). However, TSP-2-null and WT mice had similar numbers of blood vessels at 4 and 8 weeks. Detection of the tight junction proteins claudin 5 and zonula occludens protein 1 revealed similar levels of both proteins in WT and TSP-2-null vessels (data not shown). In contrast, collagen type IV deposition in blood vessels adjacent to the implant was diminished in TSP- 2-null mice (Figure 5). Because there are an equal number of blood vessels in TSP-2-null and WT mice at this point, we conclude that the reduction in collagen type IV immunoreactivity is indicative of abnormal basement membranes in TSP-2-null mice.

\section{Enhanced Expression of MMP-9 and MMP-2 in TSP-2 KO Mice}

Based on multiple studies indicating an association between the levels of TSP-2 and MMP-2 and MMP-9,
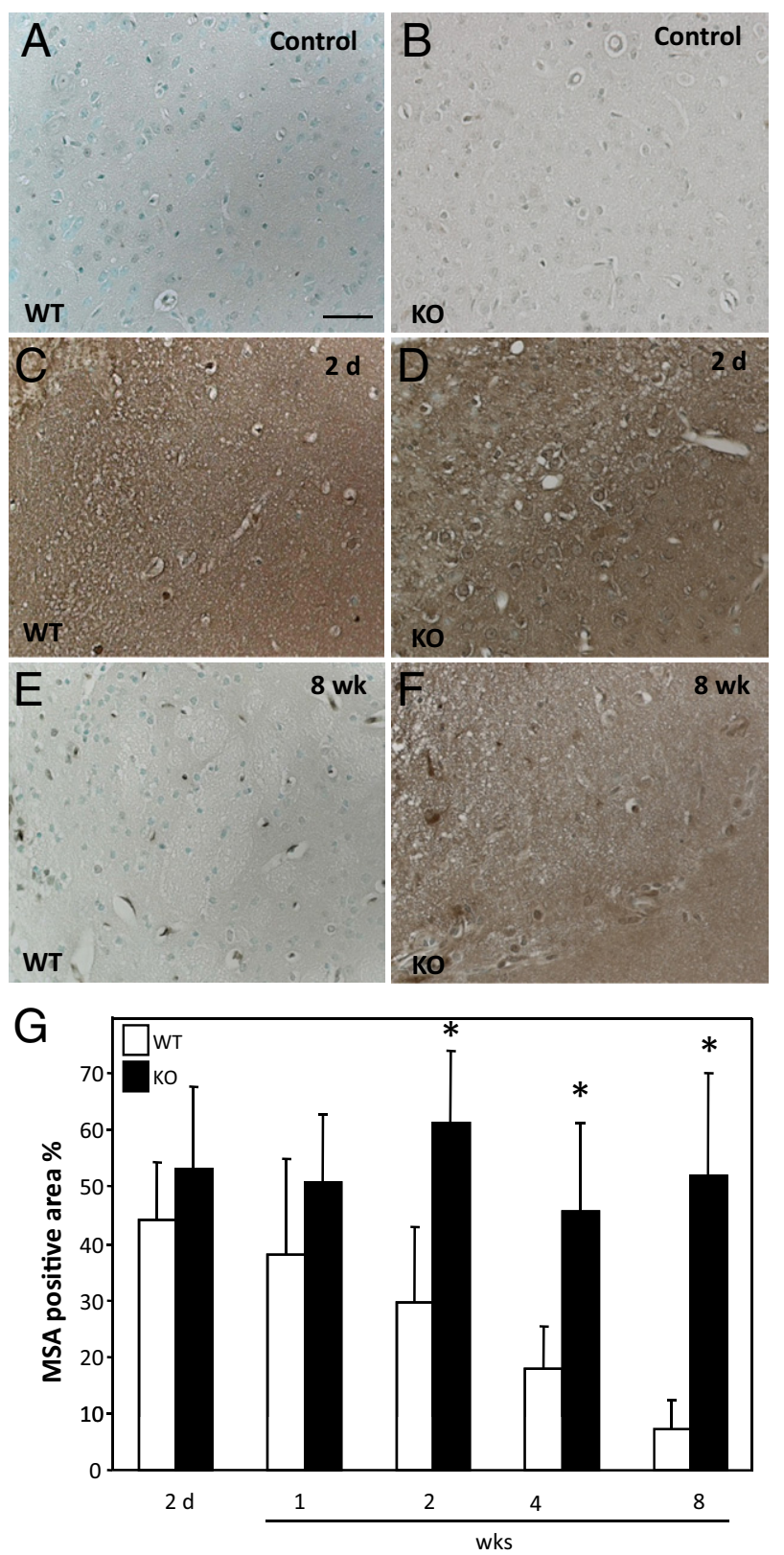

Figure 4. The presence of MSA in tissue sections indicates BBB leakage. WT sections (A, C, and $\mathbf{E}$ ) showed that BBB leakage peaked immediately after implantation and resolved over 8 weeks. Sections from TSP-2 KO mice (B, D, and $\mathbf{F}$ ) showed persistent BBB leakage. G: Histomorphometric analysis of MSA revealed increased and persistent levels in TSP-2 KO mice. Scale bar $=$ $100 \mu \mathrm{m} .{ }^{*} P<0.05$. 
A

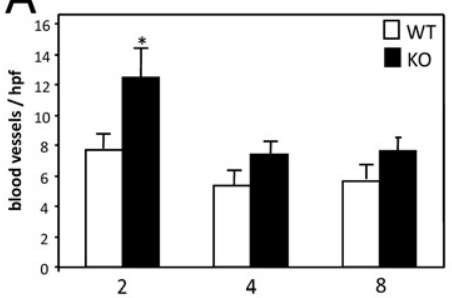

B
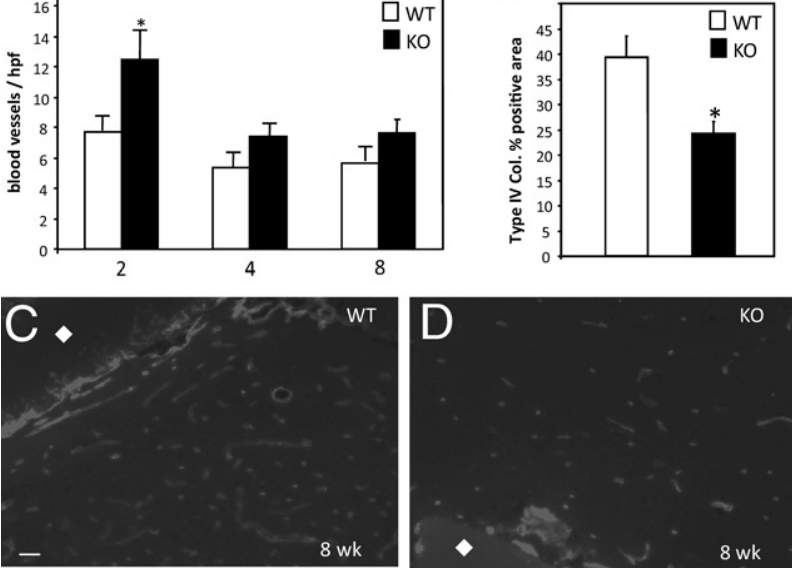

Figure 5. Blood vessel density in the FBR. A: Sections from TSP-2 KO mice showed an increase in blood vessels at 2 weeks after implantation. B: The reduced collagen (Col.) type IV in TSP-2 KO tissue implies basement membrane degradation. C and D: Similar sections from TSP-2 KO mice were stained for the presence of Col. type IV at 8 weeks. Scale bar $=100 \mu \mathrm{m}$. ${ }^{*} P<$ 0.05 (A and B). Diamonds indicate implant (C and $\mathbf{D})$. hpf, high-power field

we postulated that changes in the levels of these MMPs could be associated with reduced collagen type IV levels and the leakage of the BBB. In addition, previous studies $^{3,19,20}$ have shown that MMP-2 and MMP-9 are involved in the disruption of the BBB in a variety of models. Detection of MMPs by in situ zymography and analysis of brain extracts by gel zymography revealed that gelatinase activity was elevated in TSP-2-null mice (Figure 6). In addition, analysis of brain extracts by gel zymography at 4 weeks showed elevated levels of both MMP-2 and MMP-9 in TSP-2 KO tissue. In fact, in WT extracts, only MMP-2 was detected. To examine the importance of MMP-2 and MMP-9 in the repair of the $\mathrm{BBB}$, we treated mice with the gelatinase inhibitor 2 weeks after the implantation of filters. Mice received i.p. injections every 3 days for 2 weeks, and the integrity of the BBB was analyzed. All mice displayed excessive leakage of the BBB similar to that observed in TSP-2 KO mice, suggesting that MMPs are required for normal repair (data not shown).

\section{TSP-2 Deficiency and Endothelial Barrier Formation and Function}

An astrocyte-mBMEC transwell co-culture model was used to investigate the EC barrier formation and repair in vitro. Astrocytes derived from WT or TSP-2-null mice were co-cultured with WT mBMECs, and electrical resistances were measured for 5 days. The transepithelial electrical resistance of the co-culture with either WT or TSP-2-null astrocytes increased steadily for the duration of the experiment, and no differences between the two groups were observed (Figure 7). The inclusion of the MMP inhibitor prevented the increase in transepithelial electrical resistance in BMECs co-cultured with either TSP-2-null (Figure 7) or WT (data not shown) astrocytes. Thus, we concluded that barrier formation is dependent on gelatinase activity. To check barrier permeability, Evans blue dye was added to the upper chamber of the co-cultures on day 5 and subsequently detected in the bottom chamber at 15 minutes by spectroscopy. mBMEC monolayers co-cultured with either WT or TSP-2-null astrocytes restricted the flow of Evans blue dye into the bottom chamber to an equal extent, suggesting that TSP-2 deficiency does not influence the formation of an endothelial barrier in vitro [OD, $600 \mathrm{~nm}$; (mean \pm SEM) $0.038 \pm 0.008$ for WT and $0.04 \pm 0.009$ for $\mathrm{KO} ; n=9]$. In contrast, mBMECs co-cultured with WT or KO astrocytes in the presence of the MMP inhibitor showed significant levels of Evans blue dye in the bottom chamber [OD, 600 $\mathrm{nm} ;($ mean \pm SEM) $0.21 \pm 0.05$ for WT and $0.19 \pm 0.04$ for $\mathrm{KO} ; n=6$ ].

To simulate barrier disruption, established co-cultures with intact barrier function were treated with LPS and subjected to the Evans blue dye permeability assay. At 5 minutes after LPS exposure, dye was detected in the bottom chambers of both groups but was significantly higher in co-cultures containing TSP-2 KO astrocytes. Between 5 and 15 minutes, co-cultures of WT astrocytes and mBMECs displayed a progressive decrease in the flow of dye to the bottom chamber and reached baseline values that were indicative of complete repair. In contrast, TSP-2 KO astrocyte-mBMEC
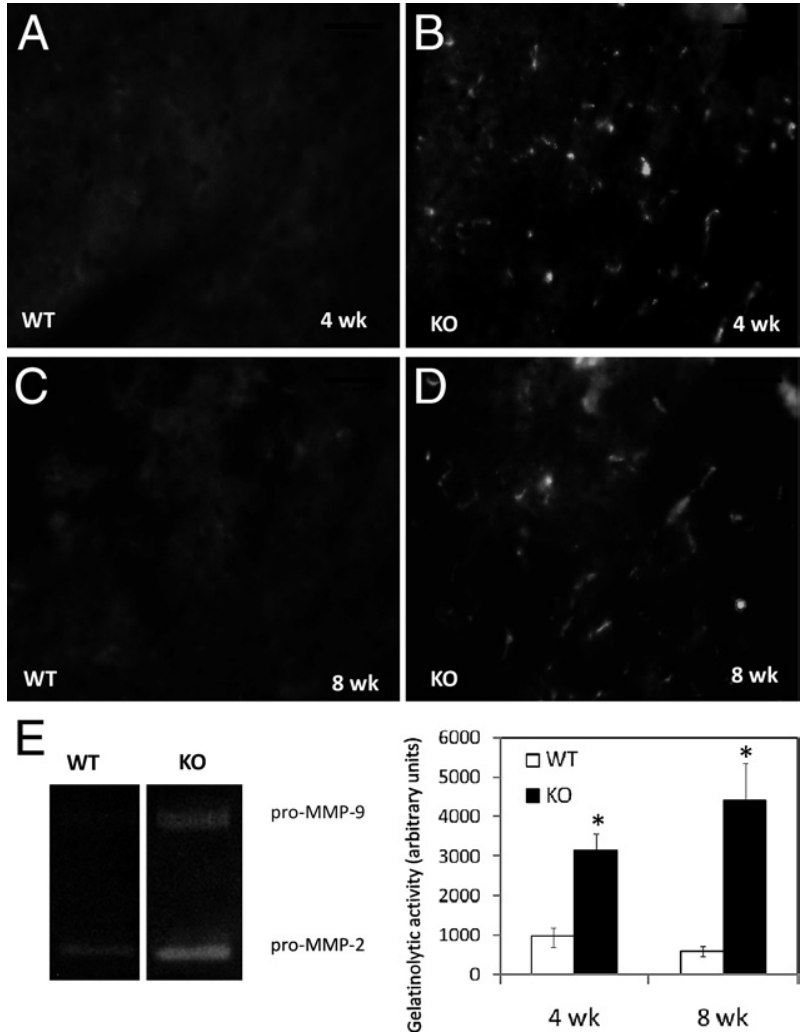

Figure 6. Elevated levels of MMPs in TSP-2 KO mice. Immunofluorescent detection shows elevated MMP-2 and MMP-9 in TSP-2 KO tissue (B and D when compared with WT tissue (A and $\mathbf{C}$ ) at 4 and 8 weeks after implantation. Zymography of 4-week brain extracts revealed both MMP-9 and MMP-2 in KO tissue but only MMP-2 in WT tissue (image is from a single experiment, with additional lanes removed for clarity). E: By using software to extract intensity data from in situ zymography, images revealed significant $\left({ }^{*} P<\right.$ 0.001 ) increases in MMP activity in KO mice at 4 and 8 weeks. 

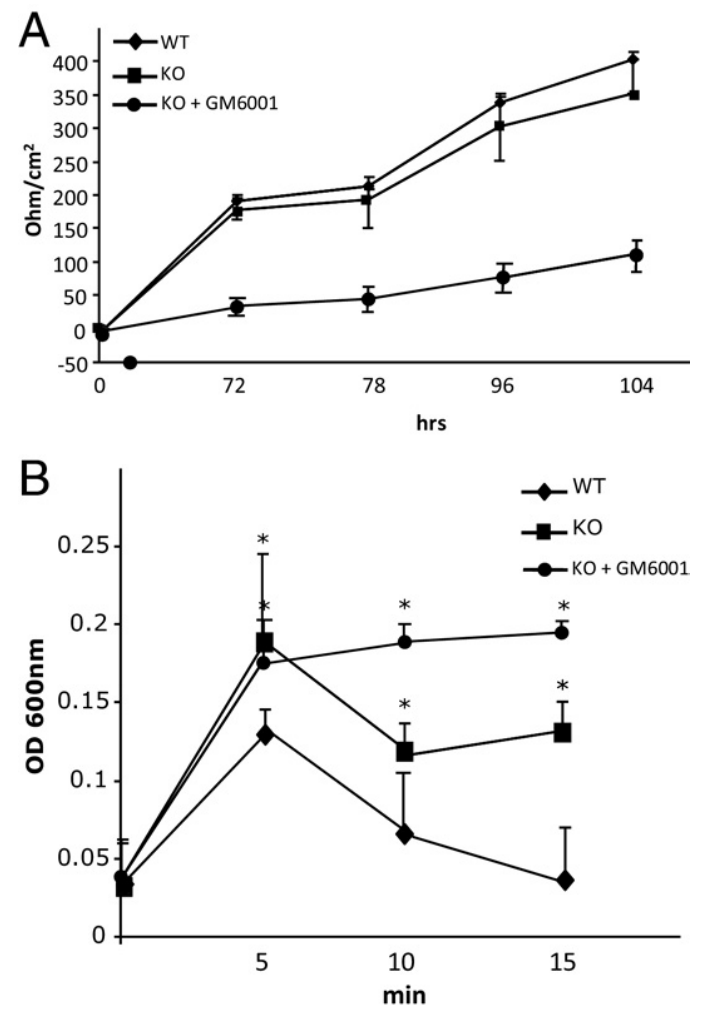

C

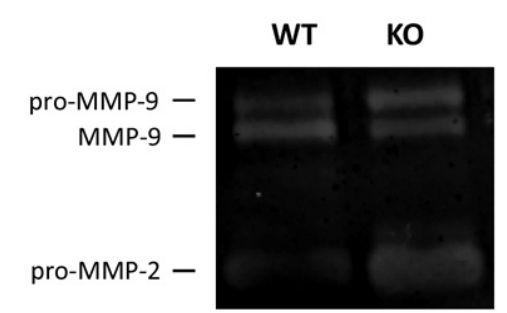

Figure 7. TSP-2 KO astrocytes do not support BBB repair. Astrocytes were isolated from either WT or TSP-2 KO mice with co-cultured mBMECs on opposite sides of a transwell membrane. A: Resistance measurements showed TSP-2 KO cells were able to form a barrier. In addition, inclusion of the MMP inhibitor prevented barrier formation. LPS was added to disrupt the formed barrier and followed with the addition of Evans blue dye. B: WT co-cultures were able to reform the barrier and exclude the Evans blue dye (measured by OD at $600 \mathrm{~nm}$ ), whereas TSP-2 KO cells did not quickly reform the barrier. Inclusion of the MMP inhibitor prevented the restoration of barrier function. C: When the isolated astrocytes were stimulated with LPS, zymography revealed higher levels of MMP-2 in TSP-2 KO cells. ${ }^{*} P<0.05$. GM6001 indicates MMP inhibitor

co-cultures displayed prolonged and increased presence of dye in the bottom chambers, even at 15 minutes, suggesting that TSP-2 deficiency negatively influences restoration of endothelial barrier function (Figure 7). Moreover, inclusion of the MMP inhibitor led to persistent loss of barrier function, suggesting that in this in vitro model, BBB repair requires MMPs. Zymographic analysis of the conditioned media of isolated astrocytes, stimulated with LPS, revealed higher levels of MMP-2 in TSP-2-null cells.

\section{Discussion}

In the present study, we investigated the role of TSP-2 in the FBR and the repair of the BBB by analyzing the differences in response to a mixed cellulose ester cortical implant between WT and TSP-2-null mice. Intracorporeal implanted biomaterials and medical devices induce an FBR that consists of a series of overlapping phases, including inflammation, foreign body giant cell formation, and formation of a collagenous capsule that surrounds the implant. ${ }^{1}$ In the brain, the features of the FBR include astroglial scarring, chronic inflammation, and neural cell death. ${ }^{2}$ We previously used a cortical polyvinyl alcohol implant that allowed us to implicate TSP-2 in the brain FBR. Specifically, we observed changes in TSP-2-null mice suggestive of a role of TSP-2 in ECM remodeling, angiogenesis, and BBB repair. ${ }^{10}$ This study was conducted using an improved implant model and in vitro studies with primary brain ECs and astrocytes. The cellulose ester material induced a normal FBR and was sectioned with the surrounding tissue, which allowed for exceptional histological analysis. We found that TSP-2null mice exhibited increased MMP levels and activity, decreased collagen type IV in blood vessels, and prolonged leakage of the BBB. In addition, in vitro studies allowed us to examine the cellular basis of sustained BBB leakage in TSP-2-null mice. Collectively, our results indicate the unique requirement for TSP-2 during a normal astrocyte response to biomaterials.

In contrast to TSP-2, we were unable to detect induction of TSP-1 in the brain FBR. We found this observation surprising because previous studies ${ }^{21}$ have linked both TSP-1 and TSP-2 in central nervous system injury. For example, the expression of both TSP-1 and TSP-2 is increased after stroke. ${ }^{12}$ In addition, astrocyte-derived TSP-1 and TSP-2 were required for rewiring of barrel cortices after injury. ${ }^{22}$ We believe these observations highlight the importance of both TSPs in synaptic remodeling after injury. However, it is clear from the present study that the physical injury from implantation and the continuous presence of the implant appear to involve only TSP-2.

Experiments on TSP-2-null mice have shown the involvement of TSP-2 in the modulation of ECM remodeling and angiogenesis during wound healing, ischemia, and the FBR. Consistent with previous findings, our study showed that inflammation in the TSP-2-null mice is persistent after 8 weeks, in contrast to the WT mice, in which the presence of reactive astrocytes and macrophages/ microglia decreased significantly after 4 weeks. Several studies $^{23}$ have investigated the roles of astrocytes in the formation and maintenance of the BBB. Astrocytes participate in the formation of the BBB by inducing capillary and venule ECs in the central nervous system to form the BBB. ${ }^{24}$ The intercellular communication between astrocytes and ECs induces the formation of the tight junctions that generate the BBB. Astrocytic processes elongate their end feet to isolate the brain parenchyma from the vascular compartment and establish the BBB. ${ }^{25}$ Furthermore, the CD95 receptor, expressed by astrocytes, induces CD95L-mediated degeneration under inflammatory conditions, which may lead to the breakdown of astrocyte end feet and the disruption of the BBB. ${ }^{26}$

Our previous observations ${ }^{10}$ using polyvinyl alcohol sponges suggested that astrocytes were a major source of TSP-2. Herein, we confirmed this suggestion by isolat- 
ing and analyzing primary astrocytes. In addition, the presence of sustained GFAP immunoreactivity and high levels of reactive microglia/macrophages confirmed that the astrocyte reaction and inflammation were enhanced in TSP-2-null mice when compared with WT mice. Reactive astrocytes were detected from 2 days to 8 weeks, and the levels were significantly higher in the TSP-2-null mice when compared with the WT mice.

The detection of MSA in brain histological features indicates the leakiness of the BBB and suggests a pathological condition. We observed elevated levels of MSA in the TSP-2 KO brain 8 weeks after the biomaterialinduced injury, suggesting the prolonged presence of a leaky BBB. Increased vascular leakage has also been observed in TSP-2-null mice during skin inflammation. ${ }^{8}$ Collagen type IV deposition in blood vessels adjacent to the implants was diminished in TSP-2-null mice. Collagen type IV is a component of the ECM and is degraded by MMPs. The reduced collagen implies basement membrane degradation, likely mediated by an increase in MMP-2 and MMP-9, which results in BBB leakage and MSA extravasation. Previous studies ${ }^{20}$ have shown that MMP-9 can degrade the main components of basal lamina in humans, including collagen type IV, after an ischemic stroke. Furthermore, leukocyte-derived MMP-9 in the ischemic brain contributes to the loss of BBB integrity by collagen type IV proteolysis. ${ }^{19}$ Despite the elevated levels of MMPs, we were unable to rescue the leakage in TSP-2 KO mice by the administration of the MMP inhibitor. Alterations in the levels of MMPs can have multiple roles in the central nervous system and have influenced BBB repair in a variable manner. For example, BBB permeability was reduced by MMP inhibitors in several pathological conditions. ${ }^{27,28}$ In addition, in a stroke model, early and late inhibition of MMPs resulted in neuroprotection and increased stroke volume, respectively. ${ }^{29}$ Based on these observations and our current findings, we postulate that MMP activity is required for BBB repair and is not a suitable target for reducing leakage. This suggestion is also consistent with a previous study ${ }^{30}$ demonstrating that the MMP inhibitor could not reduce LPSinduced BBB leakage.

Our results suggest that TSP-2 modulates the levels of MMP-2 and MMP-9 levels and that the levels of these gelatinases increase in the absence of TSP-2. ${ }^{31-34}$ We suggest that this increase is associated with the degradation of collagen type IV, which disrupts the tightly packed basal lamina and hinders BBB repair. This is evidenced by the macrophages/microglia that are present at 8 weeks after implantation and the extravasation of MSA. Taken together, these observations demonstrate the critical role of TSP-2 in the brain FBR and suggest a critical role for astrocytes in the repair of the BBB during the FBR.

\section{References}

1. Polikov VS, Tresco PA, Reichert WM: Response of brain tissue to chronically implanted neural electrodes. J Neurosci Methods 2005, 148:1-18

2. McConnell GC, Rees HD, Levey Al, Gutekunst CA, Gross RE, Bellamkonda RV: Implanted neural electrodes cause chronic, local in- flammation that is correlated with local neurodegeneration. J Neural Eng 2009, 6:056003

3. Tian W, Kyriakides TR: Matrix metalloproteinase- 9 deficiency leads to prolonged foreign body response in the brain associated with increased IL-1beta levels and leakage of the blood-brain barrier. Matrix Biol 2009, 28:148-159

4. Kyriakides TR, Maclauchlan S: The role of thrombospondins in wound healing, ischemia, and the foreign body reaction. J Cell Commun Signal 2009, 3:215-225

5. Bornstein P: Thrombospondins function as regulators of angiogenesis. J Cell Commun Signal 2009, 3:189-200

6. Maclauchlan S, Skokos EA, Agah A, Zeng J, Tian W, Davidson JM Bornstein $\mathrm{P}$, Kyriakides TR: Enhanced angiogenesis and reduced contraction in thrombospondin-2-null wounds is associated with increased levels of matrix metalloproteinases-2 and -9 , and soluble VEGF. J Histochem Cytochem 2009, 57:301-313

7. Kyriakides TR, Hartzel T, Huynh G, Bornstein P: Regulation of angiogenesis and matrix remodeling by localized, matrix-mediated antisense gene delivery. Mol Ther 2001, 3:842-849

8. Lange-Asschenfeldt B, Weninger W, Velasco P, Kyriakides TR, von Andrian UH, Bornstein P, Detmar M: Increased and prolonged inflammation and angiogenesis in delayed-type hypersensitivity reactions elicited in the skin of thrombospondin-2-deficient mice. Blood 2002, 99:538-545

9. Chen J, Somanath PR, Razorenova O, Chen WS, Hay N, Bornstein P Byzova TV: Akt1 regulates pathological angiogenesis, vascular maturation and permeability in vivo. Nat Med 2005, 11:1188-1196

10. Tian W, Kyriakides TR: Thrombospondin 2-null mice display an altered brain foreign body response to polyvinyl alcohol sponge implants. Biomed Mater 2009, 4:015010

11. Schroers J, Kumar G, Hodges TM, Chan S, Kyriakides TR: Bulk metallic glasses for biomedical applications. JOM 2009, 61:21-29

12. Lin TN, Kim GM, Chen JJ, Cheung WM, He YY, Hsu CY: Differential regulation of thrombospondin-1 and thrombospondin-2 after focal cerebral ischemia/reperfusion. Stroke 2003, 34:177-186

13. Christopherson KS, Ullian EM, Stokes CC, Mullowney CE, Hell JW, Agah A, Lawler J, Mosher DF, Bornstein P, Barres BA: Thrombospondins are astrocyte-secreted proteins that promote CNS synaptogenesis. Cell 2005, 120:421-433

14. van Vliet EA, da Costa Araujo S, Redeker S, van Schaik R, Aronica E, Gorter JA: Blood-brain barrier leakage may lead to progression of temporal lobe epilepsy. Brain 2007, 130:521-534

15. Zhang JW, Gottschall PE: Zymographic measurement of gelatinase activity in brain tissue after detergent extraction and affinity-support purification. J Neurosci Methods 1997, 76:15-20

16. Krady MM, Zeng J, Yu J, Maclauchlan S, Skokos EA, Tian W, Bornstein P, Sessa WC, Kyriakides TR: Thrombospondin-2 modulates extracellular matrix remodeling during physiological angiogenesis. Am J Pathol 2008, 173:879-891

17. Stamatovic SM, Shakui P, Keep RF, Moore BB, Kunkel SL, Van Rooijen N, Andjelkovic AV: Monocyte chemoattractant protein-1 regulation of blood-brain barrier permeability. J Cereb Blood Flow Metab 2005, 25:593-606

18. Hertz L, Schousboe A, Boechler N, Mukerji S, Fedoroff S: Kinetic characteristics of the glutamate uptake into normal astrocytes in cultures. Neurochem Res 1978, 3:1-14

19. Gidday JM, Gasche YG, Copin JC, Shah AR, Perez RS, Shapiro SD, Chan PH, Park TS: Leukocyte-derived matrix metalloproteinase-9 mediates blood-brain barrier breakdown and is proinflammatory after transient focal cerebral ischemia. Am J Physiol Heart Circ Physiol 2005, 289:H558-H568

20. Rosell A, Cuadrado E, Ortega-Aznar A, Hernandez-Guillamon M, Lo $\mathrm{EH}$, Montaner J: MMP-9-positive neutrophil infiltration is associated to blood-brain barrier breakdown and basal lamina type IV collagen degradation during hemorrhagic transformation after human ischemic stroke. Stroke 2008, 39:1121-1126

21. Eroglu C: The role of astrocyte-secreted matricellular proteins in central nervous system development and function. J Cell Commun Signal 2009, 3:167-176

22. Eroglu C, Allen NJ, Susman MW, O'Rourke NA, Park CY, Ozkan E Chakraborty C, Mulinyawe SB, Annis DS, Huberman AD, Green EM, Lawler J, Dolmetsch R, Garcia KC, Smith SJ, Luo ZD, Rosenthal A, Mosher DF, Barres BA: Gabapentin receptor alpha2delta-1 is a neu- 
ronal thrombospondin receptor responsible for excitatory CNS synaptogenesis. Cell 2009, 139:380-392

23. Ballabh $\mathrm{P}$, Braun $\mathrm{A}$, Nedergaard M: The blood-brain barrier: an overview: structure, regulation, and clinical implications. Neurobiol Dis 2004, 16:1-13

24. Janzer RC, Raff MC: Astrocytes induce blood-brain barrier properties in endothelial cells. Nature 1987, 325:253-257

25. Bechmann I, Galea I, Perry VH: What is the blood-brain barrier (not)? Trends Immunol 2007, 28:5-11

26. Becher B, Barker PA, Owens T, Antel JP: CD95-CD95L: can the brain learn from the immune system? Trends Neurosci 1998, 21:114-117

27. Sood RR, Taheri S, Candelario-Jalil E, Estrada EY, Rosenberg GA: Early beneficial effect of matrix metalloproteinase inhibition on bloodbrain barrier permeability as measured by magnetic resonance imaging countered by impaired long-term recovery after stroke in rat brain. J Cereb Blood Flow Metab 2008, 28:431-438

28. Yang Y, Estrada EY, Thompson JF, Liu W, Rosenberg GA: Matrix metalloproteinase-mediated disruption of tight junction proteins in cerebral vessels is reversed by synthetic matrix metalloproteinase inhibitor in focal ischemia in rat. J Cereb Blood Flow Metab 2007, 27:697-709
29. Zhao BQ, Wang S, Kim HY, Storrie H, Rosen BR, Mooney DJ, Wang $X$, Lo EH: Role of matrix metalloproteinases in delayed cortical responses after stroke. Nat Med 2006, 12:441-445

30. Rosenberg GA, Estrada EY, Mobashery S: Effect of synthetic matrix metalloproteinase inhibitors on lipopolysaccharide-induced bloodbrain barrier opening in rodents: differences in response based on strains and solvents. Brain Res 2007, 1133:186-192

31. Kyriakides TR, Zhu YH, Yang Z, Huynh G, Bornstein P: Altered extracellular matrix remodeling and angiogenesis in sponge granulomas of thrombospondin 2-null mice. Am J Pathol 2001, 159 : 1255-1262

32. Kyriakides TR, Maclauchlan S: The role of thrombospondins in wound healing, ischemia, and the foreign body reaction. J Cell Commun Signal 2009, 3:215-225

33. Agah A, Kyriakides TR, Bornstein P: Proteolysis of cell-surface tissue transglutaminase by matrix metalloproteinase-2 contributes to the adhesive defect and matrix abnormalities in thrombospondin-2-null fibroblasts and mice. Am J Pathol 2005, 167:81-88

34. Kokenyesi R, Armstrong LC, Agah A, Artal R, Bornstein P: Thrombospondin 2 deficiency in pregnant mice results in premature softening of the uterine cervix. Biol Reprod 2004, 70:385-390 